\title{
Visuo-haptic interactions in unilateral spatial neglect: the cross modal Judd illusion
}

\author{
Flavia Mancini ${ }^{1,2}{ }^{*}$, Emanuela Bricolo ${ }^{1}$, Flavia C. Mattioli ${ }^{3}$ and Giuseppe Vallar ${ }^{1,2}$ * \\ ${ }^{1}$ Department of Psychology, University of Milano-Bicocca, Milano, Italy \\ ${ }^{2}$ Neuropsychological Laboratory, IRCCS Italian Auxological Institute, Milano, Italy \\ ${ }^{3}$ Neuropsychological Unit, Spedali Civili di Brescia, Brescia, Italy
}

\section{Edited by:}

Nadia Bolognini, University

Milano-Bicocca, Italy

Reviewed by:

Tony Ro, City College of New York, USA

David Pitcher, Massachusetts Institute of Technology, USA

*Correspondence:

Flavia Mancini and Giuseppe Vallar, Department of Psychology, University of Milano-Bicocca, Piazza dell'Ateneo Nuovo 1, 20126 Milano, Italy. e-mail:f.mancini2@campus.unimib.it; giuseppe.vallar@unimib.it
Unilateral spatial neglect (USN) has been mainly investigated in the visual modality; only few studies compared spatial neglect across different sensory modalities, and explored their multisensory interactions, with controversial results. We investigated the integration between vision and haptics, through a bisection task of a cross modal illusion, the Judd variant of the Müller-Lyer illusion. We examined right-brain-damaged patients with $(n=7)$ and without $(n=7)$ left USN, and neurologically unimpaired participants $(n=14)$ in the bisection of Judd stimuli under visual, haptic, and visuo-haptic presentation. Neglect patients showed the characteristic rightward bias in the bisection of the baseline stimuli in the visual modality, but not in the haptic and visuo-haptic conditions. The illusory effects were preserved in each group and in each modality, indicating that the processing of the cross modal illusion is independent of the presence of deficits of spatial attention and representation. Spatial neglect can be modality-specific, but visual and tactile sensory inputs are properly integrated.

Keywords: unilateral spatial neglect, multisensory integration, illusion, vision, haptics, touch, somatosensory processing

\section{INTRODUCTION}

Unilateral spatial neglect (USN) is a frequent neuropsychological syndrome occurring after lesions to the right hemisphere. USN is characterized by the patients' failure to report sensory events taking place in the portion of space contralateral to the side of the lesion (contralesional), and to explore through motor acts that portion of space (Vallar, 1998; Halligan et al., 2003; Heilman et al., 2003; Husain, 2008). A rightward bias in line bisection is considered one of the signatures of USN (Bisiach et al., 1976, 1983; Schenkenberg et al., 1980), which has been mainly investigated in the visual modality, with fewer studies assessing the haptic modality, and reporting controversial results.

The aim of this study was twofold: (i) to compare the severity of left USN, as assessed by a line bisection task, in two unimodal visual, and haptic conditions, which were entirely comparable except for the availability of unisensory information; (ii) to assess the patients' ability to combine information from different sensory modalities, i.e., vision and haptics. To this aim, we used a line bisection task involving the processing of a cross modal illusion that we had previously used with neurologically unimpaired individuals (Mancini et al., 2010).

As established by motor exploratory tasks, USN may occur in both the visual and the tactile modality (De Renzi et al., 1970; Beschin et al., 1996; Haeske-Dewick et al., 1996). Evidence has however been provided to the effect that USN may be less severe and even absent in the tactile modality, in the absence of visual input. An early and seminal observation is provided by Gilliatt and Pratt (1952) about a right-brain-damaged (RBD) patient who showed severe left USN, when required to circle pins using a pencil with eyes open; conversely, with eyes closed, the patient explored the whole board up to the extreme left. A number of subsequent studies found a visuo-haptic difference, with USN being more severe in the visual modality for spatial exploratory tasks (Chedru, 1976, in RBD patients with a visual-half-field deficit; Villardita, 1987; Gentilini et al., 1989; Schindler et al., 2006). However, in the study by Chedru (1976) RBD patients without visual half-field deficits showed a more severe USN when blindfolded. Importantly, there is evidence that the deficit may be modality-specific (Cubelli et al., 1991 re-analysis of the data of Gentilini et al., 1989; Vallar et al., 1991b). Particularly, in their re-analysis Cubelli et al. (1991) reported four RBD patients who showed a disproportionate rightward bias with open eyes, but not with eyes closed, in a task requiring to explore a keyboard; three patients showed the opposite pattern (rightward bias with eyes closed), while five patients were impaired in both conditions. Other studies found a double dissociation between visual and tactile USN, reporting patients with a defective performance either in the visual or in the tactile modality (Perani et al., 1987, Appendix 2; Barbieri and De Renzi, 1989; Vallar et al., 1991b).

In line bisection tasks, the available studies indicate that the rightward bias appears to be confined to the visual modality (Fujii et al., 1991; Hjaltason et al., 1993; Chokron et al., 2002). In haptic bisection, no rightward bias has been found, with left USN being almost absent (for a review, see Brozzoli et al., 2006; Gainotti, 2010). Taken together, these findings indicate that USN may be more severe in the visual than in the tactile modality. The deficits 
may also be modality-specific in exploratory tasks, conjuring up a double dissociation (Vallar, 2000) between vision and touch, and suggesting the existence of modality-specific attentional and representational components (Vallar, 1998). In line with this idea, a rehabilitation study showed that a 6-weeks visual attention training improved visual but not tactile detection of left-sided targets (Làdavas et al., 1994). Finally, it is also well-known that visual and tactile extinction to double simultaneous stimulation may occur independently of each other after unilateral brain damage (Vallar et al., 1994a; Hillis et al., 2006).

The evidence for modality-specificity, currently framed in the broader context of a multi-componential attentional/representational account of the USN deficit (Barbieri and De Renzi, 1989; Vallar, 1998), may be contrasted with an early interpretation of the syndrome in terms of a higher-order sensory impairment, hypothesized as a defective "spatial summation" and termed "amorphosynthesis" (with the primary function of "morphosynthesis" being the recognition of form, Denny-Brown et al., 1952). "The loss of visual components of such morphosynthesis in addition to tactile factors, is the basis of unawareness of part of extrapersonal space and unawareness of self, without disorder of the concept of space or of body schema. Such unawareness differs considerably from simple loss of sensation" (ibidem, p. 470). While the very concept of amorphosynthesis is admittedly vague, it appears to suggest the impairment of a higher-order spatial factor, related to different sensory modalities.

In our experimental task we used a well-known visual illusion, the Judd figure, that has proven to be cross modal (Mancini et al., 2011). Visual illusions are a tool for investigating implicit processing in USN, since illusory effects arising from the left side of space can be preserved and do not require perceptual awareness to occur (see Vallar and Daini, 2006, for a review). Among these, the MüllerLyer illusion consists in a line at which ends two outward- or two inward-oriented arrowheads are located. In line bisection tasks, the Judd variant of this illusion (namely, a line with two identical arrowheads at its ends; Judd, 1899; Holding, 1970) has been used (Ro and Rafal, 1996; Mancini et al., 2010). The bisection of the shaft is shifted toward the tail end under visual and haptic presentation in neurologically unimpaired participants; furthermore, the visual illusion transfers cross modally, modulating bisection in the tactile modality. The spatial correspondence between the visual and the tactile stimuli constituting the cross modal illusion has a crucial role in the cross modal transfer (Mancini et al., 2010).

Although the Müller-Lyer illusion has proven to be useful for studying implicit processing in the contralesional space in patients with left USN, these investigations have been confined to the visual modality: haptic and cross modal illusory effects have not been investigated so far (Vallar and Daini, 2006). We therefore examined right brain-damaged patients with and without left USN in the bisection of the Judd variant of the MüllerLyer illusion under visual, haptic, and visuo-haptic presentation. We aimed at assessing whether visuo-tactile interactions were preserved in these patients. The ability of RBD patients with left USN to combine visuo-haptic information could also provide an experimental assessment of the "amorphosynthesis" hypothesis.

\section{MATERIALS AND METHODS PARTICIPANTS}

Fourteen RBD patients and 14 neurologically unimpaired participants took part in the study (see Table 1). RBD patients were subdivided into two groups, with and without left USN $(\mathrm{N}+/ \mathrm{N}-)$, as assessed by a standard neuropsychological battery (Table 2). Even if a perusal of Table 1 suggests that $\mathrm{N}+$ patients may be older (as previously found in larger series of patients, Leibovitch et al., 1998; Gottesman et al., 2008), the age of the participants of the three groups was comparable, as assessed by a one-way analysis of variance $[F(2,25)=3.16, p>0.60]$. Each participant gave written informed consent to take part in the experiment, which had been approved by the Ethics Committee of the IRCSS Istituto Auxologico Italiano.

The patients' demographic and neurological data are summarized in Table 1. All 14 RBD patients had unilateral stroke lesions in the right hemisphere. All patients were right-handed, and had no history or neurological evidence of previous neurological diseases, psychiatric disorders, or dementia. All patients had a normal or corrected-to-normal vision. Contralesional motor, somatosensory, and visual half-field deficits, including extinction to tactile and visual double simultaneous stimuli, were assessed by a standard neurological exam (Bisiach and Faglioni, 1974). Visual field defects were also assessed by kinetic Goldmann perimetry, and by a computerized program testing six different positions in both the left and right hemi-fields, at different eccentricities $\left(3^{\circ}, 6^{\circ}, 12^{\circ}\right)$. The visual field of two $\mathrm{N}-$ patients (P10 and P14) was tested with our customized program only.

The lesion site and size were assessed by CT or MRI scan. Lesions were mapped for each RBD patient using the MRIcro software (Rorden and Brett, 2000) and were drawn manually onto selected horizontal slices of a standard template brain. Figure 1 shows the overlapped lesion maps of the 14 RBD patients, separately for patients with and without left USN. In N+ patients the maximum overlap involved the right putamen, insula, and frontal inferior orbital cortices (seven patients); in $\mathrm{N}-$ patients the maximum overlap was observed over the right rolandic operculum, the superior temporal pole, and the insula (three patients). Overall, lesions were more extensive in the $\mathrm{N}+$ group (mean volume of the lesion $=126 \mathrm{cc}, \mathrm{SD} \pm 79.51$, range $74.44-282.76 \mathrm{cc}$ ) than in the $\mathrm{N}-$ group (mean volume of the lesion $=41.04 \mathrm{cc}, \mathrm{SD} \pm 54.24$, range $1.22-129.16 \mathrm{cc}$ ), in line with previously reported evidence in large series of patients (Hier et al., 1983a,b; Leibovitch et al., 1998).

\section{Baseline neuropsychological assessment}

The diagnostic battery assessing the presence of left USN included three visuomotor exploratory tasks (line, letter, and bell cancelation), a reading task, a line bisection task, two copying tasks, and one drawing from memory task (Table 2). Patients used their right unaffected hand to perform the tasks. In all tasks, the center of the sheet was aligned with the mid-sagittal plane of the patient's trunk. The Mini Mental State Examination (MMSE) was also given (Grigoletto et al., 1999).

i. Line bisection. The patients' task was to mark with a pencil the mid-point of six horizontal black lines (two $10 \mathrm{~cm}$, two 
Table 1 | Demographical and neurological data of 14 right-brain-damaged patients with $(\mathrm{N}+$ ) and without (N-) USN, and of 14 control (C) neurologically unimpaired participants.

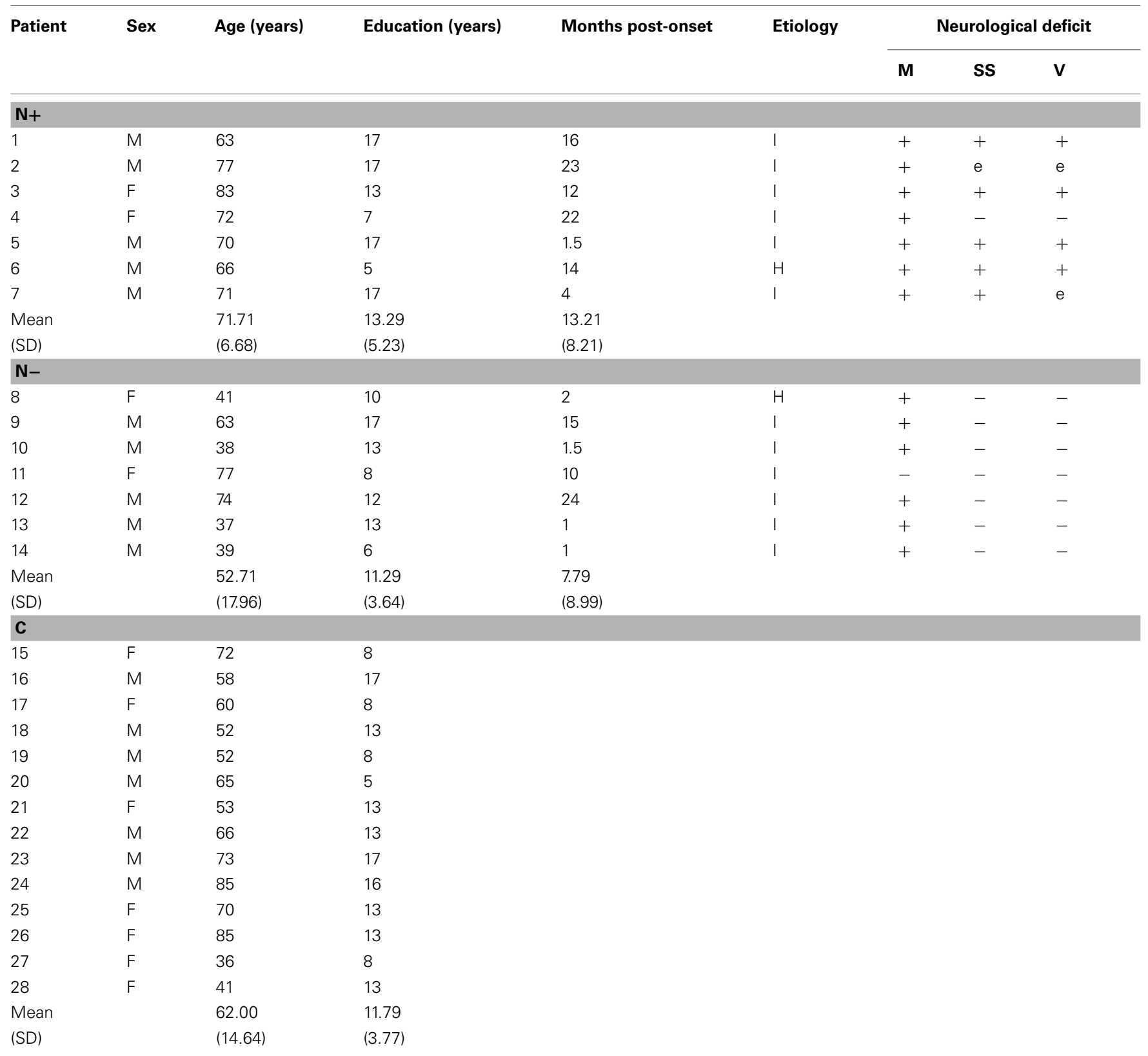

M/SSN: left motor/somatosensory/visual half-field deficits. e: Contralesional extinction.

\pm Presence/absence of impairment.

$\mathrm{M} / \mathrm{F}$, male/female; $\mathrm{I} / \mathrm{H}$, ischemic/hemorrhagic.

$15 \mathrm{~cm}$, and two $25 \mathrm{~cm}$ in length, all $2 \mathrm{~mm}$ in width), presented in a random fixed order. Each line was printed at the center of an A4 sheet. The length of the left-hand side of the line (i.e., from the left end of the line to the participant's mark) was measured to the nearest millimeter. This measure was converted into a standardized score (percentage deviation), namely: (measured left half minus objective half)/objective half $\times 100$ (Rode et al., 2006b). This transformation yields positive numbers for marks placed to the right of the objective physical center, negative numbers for marks placed to the left of it. The mean percentage deviation score of 65 neurologically unimpaired participants, matched for age ( mean $=72.2$, $\mathrm{SD} \pm 5.16$, range 65-83), and years of education ( mean $=9.5$, $\mathrm{SD} \pm 4.48$, range $5-18$ ) was $1.21 \%$ (SD \pm 3.48 , range $-16.2 \%$ to $+6.2 \%$ ). A percentage deviation score higher than 8.20 was considered as indicative of left USN (Fortis et al., 2010).

ii. Line cancellation (Albert, 1973). The participants' task was to cross out all of the 21 black lines printed on an A4 sheet 
Table 2 | Clinical data of 14 right-brain-damaged patients with $(\mathrm{N}+)$ and without $(\mathrm{N}-)$ USN.

\begin{tabular}{|c|c|c|c|c|c|c|c|c|c|c|c|c|}
\hline \multirow[t]{2}{*}{ Patient } & \multirow[t]{2}{*}{ MMSE } & \multicolumn{3}{|c|}{ Cancellation tests } & \multirow[t]{2}{*}{$\begin{array}{l}\text { Line } \\
\text { bisection (\%) }\end{array}$} & \multirow[t]{2}{*}{$\begin{array}{l}\text { Sentence } \\
\text { reading }\end{array}$} & \multicolumn{3}{|c|}{ Drawing } & \multicolumn{2}{|c|}{$\begin{array}{l}\text { Corsi's block } \\
\text { tapping test }\end{array}$} & \multirow[t]{2}{*}{$\begin{array}{l}\text { Tactile form } \\
\text { recognition }\end{array}$} \\
\hline & & Line & Letter & Bells & & & Daisy & Complex & Clock & Standard & Vertical & \\
\hline \multicolumn{13}{|l|}{$\mathrm{N}+$} \\
\hline 1 & 30 & 0 & 2 & 1 & $+11.4^{*}$ & 6 & 2 & $5.5^{*}$ & 11 & $2.75^{*}$ & 3 & 30 \\
\hline 2 & 29 & 0 & $20^{*}$ & $16^{*}$ & +3.8 & 6 & 1.5 & 10 & 12 & 3.75 & 2 & 25 \\
\hline 4 & 27 & $2^{*}$ & -1 & 3 & $+10.2^{*}$ & 6 & 2 & $9 *$ & 11 & 3.5 & 2 & $20^{*}$ \\
\hline 5 & 29 & 0 & $36^{*}$ & $7^{*}$ & +1.1 & 6 & 1.5 & $4.5^{*}$ & $6^{*}$ & $1.75^{*}$ & $1 *$ & $16^{*}$ \\
\hline 6 & 28 & $7^{*}$ & $34^{*}$ & $6^{*}$ & $+50.4^{*}$ & $0^{*}$ & 1.5 & $4^{*}$ & $8^{*}$ & $2.25^{*}$ & 2 & $14^{*}$ \\
\hline 7 & 28 & $1^{*}$ & $32^{*}$ & $7^{*}$ & +2.4 & $4^{*}$ & 2 & $5^{*}$ & 12 & 3.75 & 2 & 25 \\
\hline \multicolumn{13}{|l|}{ N- } \\
\hline 8 & 30 & 0 & 0 & 0 & +4.4 & 6 & 2 & 10 & 12 & 3.75 & 2 & 28 \\
\hline 12 & 27 & 0 & -1 & -2 & -5.6 & 6 & 2 & 10 & 12 & 4 & 3 & $22^{*}$ \\
\hline 13 & 28 & 0 & 0 & 1 & +0.8 & 6 & 2 & 10 & 12 & 3.5 & 4 & 36 \\
\hline 14 & n.a. ${ }^{1}$ & 0 & 1 & 4 & +0.2 & 6 & 2 & 10 & 11 & 4 & 4 & 34 \\
\hline
\end{tabular}

Target cancellation: numbers of left- minus right-hand-side omissions. Line bisection: percent displacement ( \pm rightward/leftward). Sentence reading, drawing tests, Corsi's block tapping tests, tactile form recognition: number of correct responses (for Corsi's standard tapping test, adjusted scores). n.a.: not assessed. ${ }^{1}$ Arabian patient. " defective performance.

with no distracters. The score was the difference between numbers of omissions in the left- (range 0-11) and in the right- (range 0-10) hand-sides of the sheet. Neurologically unimpaired participants perform this task without errors.

iii. Letter cancellation (Diller and Weinberg, 1977). The participants' task was to cross out all of $104 \mathrm{H}$ letters (53 in the left-hand-side, and 51 in the right-hand-side of the sheet), printed on an A3 sheet, together with 208 letter distracters. In neurologically unimpaired participants the maximum difference between omission errors on the two sides of the sheet is two (Vallar et al., 1994b).

iv. The Bells test (Gauthier et al., 1989). The participants' task was to cross out all of 35 bells (15 in the left-hand-side, 5 in the middle, and 15 in the right-hand-side of the sheet), printed on an A3 sheet, together with other 280 distracters. In neurologically unimpaired participants the maximum difference between omission errors on the two sides of the sheet is four (Vallar et al., 1994b).

v. Sentence reading (Pizzamiglio et al., 1992). Six sentences of different lengths were presented one at time, printed centrally on a A4 sheet. The score was the number of correctly read sentences (range 0-6). Normal participants and patients with right brain damage without USN make no errors on this test. RBD patients with USN make omission errors, substitution errors, or both, in the left half of the sentence.

vi. Drawing. Patients were required to copy two figures [a daisy and a complex figure with two trees in the left-hand-side, two pine trees in the right-hand-side, and a house in the center of an A4 sheet (Gainotti et al., 1972)], and to draw from memory the hours of a clock in a circular quadrant (diameter $12 \mathrm{~cm}$ ), printed on an A4 sheet. Omission errors were calculated as follows:

a. Daisy (range 0-2): 2 (flawless copy); 1.5 (partial omission of the left-hand-side of the daisy); 1.0 (complete omission of the left-hand-side of the daisy); 0.5 (complete omission of the left-hand-side of the daisy, and partial omission of the right-hand-side of the daisy); 0 (no drawing, or no recognizable element). The mean omission score of 148 neurologically unimpaired participants (mean age $=61.89$, $\mathrm{SD} \pm 11.95$, range $40-89)$ was $1.99(\mathrm{SD} \pm 0.12$, range $1-2)$. Accordingly, the presence of a partial or complete omission of the left-hand side of the daisy (score lower than 1.5) was considered as indicative of left USN.

b. Five-element complex drawing (range 0-10): 2 (for each flawless copied element); 1.5 (for each partial left-sided omission of one component, e.g., some branches of the left-hand-side of a tree); 1.0 (for each complete left-handside omission of one component); 0.5 (for each complete omission of the left-hand-side, and partial omission of the right-hand-side of the component); 0 (no drawing, or no recognizable element). The horizontal ground line was not considered for scoring. The mean score of 148 neurologically unimpaired participants (mean age $=61.89$, $\mathrm{SD} \pm 11.95$, range $40-89$ ) was 9.89 ( $\mathrm{SD} \pm 0.23$, range 9.5-10). Accordingly, a score lower than 9.5 indicated a defective performance.

c. Clock drawing from memory (range 0-12): 1 (for each element in the correct position); 0 (for each omission or translocation of an element from one side to the 


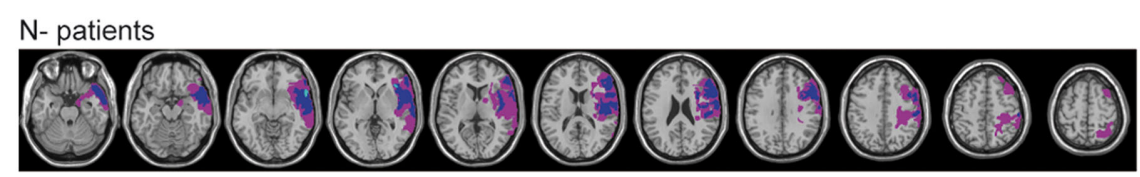

$\mathrm{N}+$ patients
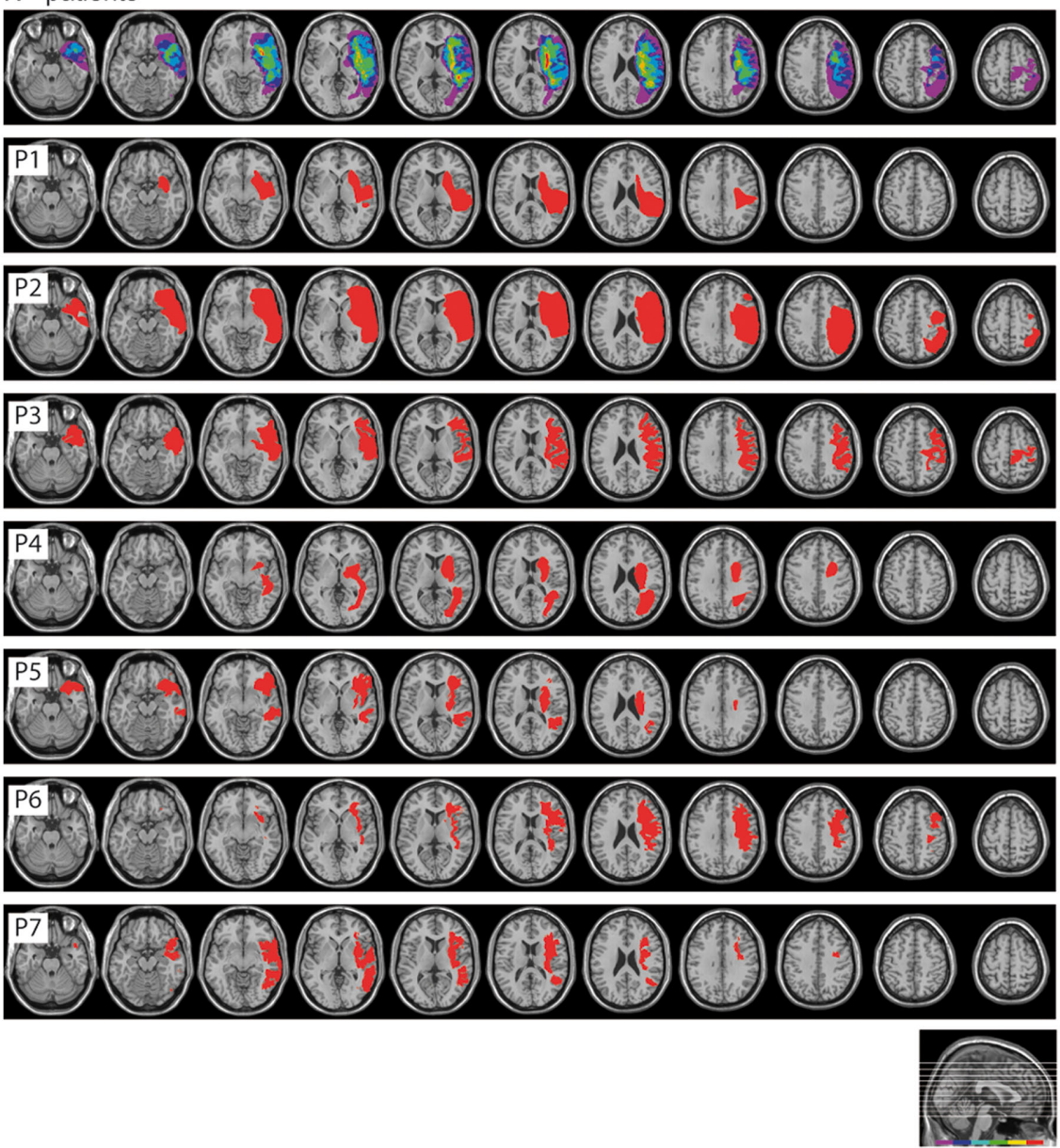

FIGURE 1 | Lesion site of patients with and without USN. Superimposed overlapping brain lesions (first two rows), and lesional mapping for each USN patient (P1-P7). The lesions were mapped using MRIcro software (http://www.sph.sc.edu/comd/rorden/mricro.html).

other; elements " 12 " and " 6 " were scored as translocated when displaced in the right- or left-hand-side quadrants). The mean score of 148 neurologically unimpaired participants (mean age $=61.89, \mathrm{SD} \pm 11.95$, range $40-89$ ) was 11.55 ( $\mathrm{SD} \pm 1.17$, range 0-6). Accordingly, a score lower than 9 indicated a defective performance. Furthermore, neurologically unimpaired participants made no translocations.

vii. Corsi's block tapping test (Orsini et al., 1987). Nine white cubes were arranged over a 23 by $28 \mathrm{~cm}$ board. The examiner tapped sequences of increasing length in a fixed order, with the patients' task being to tap the same ordered sequence, immediately after presentation. The test continued until the patient failed at a given length (less than three out of five sequences were correctly recalled). The spatial span score was the length of the longest sequence correctly recalled. Scores adjusted for gender, age, and education were computed.

viii. Corsi's block tapping vertical test. This was a modified version of the standard Corsi's block tapping test, adapted for USN patients (Ronchi et al., 2009). Nine white cubes were arranged over a vertical board $60 \mathrm{~cm}$ high and $14 \mathrm{~cm}$ wide; the distance between each cube was $1.5 \mathrm{~cm}$. The procedure was identical to that used for the standard block tapping test of Orsini et al. (1987). Control data were provided by 14 neurologically unimpaired right-handed $C$ participants, matched for age and education (mean age 62 years, range $36-85$, mean education 11.7 years): the mean span was $3.38(\mathrm{SD} \pm 1.07$, range 2-6).

ix. Modified version of the Benton tactile form assessment (Benton, 1994). We adapted the original version in order to administer 
the test to USN patients (symmetrical stimuli, central presentation of the visual comparisons). Participants were required to match a shape, explored haptically with the right hand and out of sight, to a visual sketch of the shape to be chosen among five stimuli, printed in a vertical column on an A4 sheet. Eighteen shapes, subdivided in three sections, were presented: nine filled and nine unfilled. A score of 2 was assigned to each correct response given within $30 \mathrm{~s}$, 1 within $60 \mathrm{~s}$, and 0 for wrong or out-of-time responses. The mean score of the control group $(\mathrm{C})$ was 30.91 out of $36(\mathrm{SD} \pm 4.18$, range 26-36).

For the Benton tactile form assessment and the Corsi's block tapping vertical test, the patients' performances were compared with those of control participants by $t$ tests (Crawford and Garthwaite, 2002).

\section{STIMULI AND APPARATUS}

Stimuli and apparatus were identical to a previous study we conducted in neurologically unimpaired participants (Mancini et al., 2010). Stimuli consisted of three types of black plastic figures (Figures 2A-C): a baseline control (a horizontal rod with vertical ends), and two illusory figures (a horizontal rod with leftward outgoing/rightward ingoing fins at its ends, which brought about a leftward displacement of the shaft's perceived center; a horizontal rod with leftward ingoing/rightward outgoing fins, which brought about a rightward displacement of the shaft's perceived center).

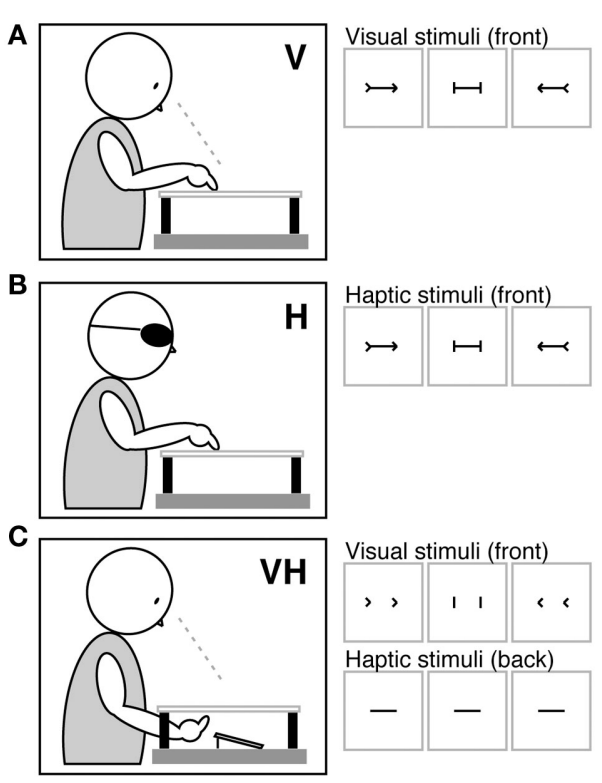

FIGURE 2 | Stimuli and apparatus. Under visual [V (A)], haptic [H (B)], and cross modal visuo-haptic [VH (C)] presentations, three types of stimuli were administered: leftward outgoing/rightward ingoing fins, which brought about a leftward displacement of the shaft's perceived center; a baseline control stimulus with vertical ends; leftward ingoing/rightward outgoing fins, which brought about a rightward displacement of the shaft's perceived center. In the visuo-haptic condition (C) the ends were glued on the front of the board, and the horizontal shaft to be bisected on the back, in the correspondent positions.
Each stimulus included a horizontal rod (10 or $12 \mathrm{~cm}$ long), and two identical ends, vertical (length: $25 \mathrm{~mm}$; height: $10 \mathrm{~mm}$; thickness: $1 \mathrm{~mm}$ ), or angled at $45^{\circ}$ (length of each fin: $35 \mathrm{~mm}$; height: $10 \mathrm{~mm}$; thickness: $1 \mathrm{~mm}$ ). All stimuli, both with vertical and angled ends, were $50 \mathrm{~mm}$ high. Each stimulus configuration was attached in the center of a white wooden board $(40 \mathrm{~cm} \times 40 \mathrm{~cm}$, thickness $0.8 \mathrm{~cm}$ ). Under visual and haptic presentation, both the arrowheads and the horizontal rod were glued on the front of the board (see Figures 2A,B). Conversely, in the cross modal condition the horizontal rod was positioned on the backside of the board centrally, and the arrowheads on the front-side in the correspondent positions (see Figure 2C).

The experiment was performed in a normally illuminated and quiet room with patients being comfortably seated in front of a table. Each board was presented individually, with its center aligned with the mid-sagittal plane of the participant's trunk, and placed flat on a wooden support at the height of $12 \mathrm{~cm}$ from the tabletop. In the cross modal condition, a mirror was placed on the table under the board. The mirror reflected the shaft on the backside and was seen by the experimenter only.

\section{PROCEDURE}

Participants received instructions to bisect with the index finger the horizontal shaft of each stimulus, using their right hand. The task was performed under visual, haptic, or visuo-haptic conditions of stimulus presentation. The three conditions were assessed during three separate sessions, in a counterbalanced order across participants. In the visual condition, participants received instructions to touch the mid-point of the shaft without exploring haptically the stimulus, and to close their eyes immediately after responding, while the experimenter measured their bisection error to the nearest millimeter (Figure 2A). In the haptic condition, blindfolded participants scanned the shapes (arrowheads and shafts) haptically, and then set the mid-point of the shaft with their right index finger. Each trial started with the experimenter placing the palm of the participant's open hand centrally over the stimulus. Stimuli were short enough to fit into the open hand. On each trial, participants were required to explore the entire shape before responding, with no time limits being set (Figure 2B). In the visuo-haptic condition, participants received instructions to look at the arrowheads on the front-side of the board, and simultaneously palpated with the whole hand the shaft glued on the backside of the board, and then set its mid-point using their right index finger. Participants did not see their right forearm, which was covered by the wooden support (Figure 2C).

In all presentation conditions (visual, haptic, visuo-haptic) the two lengths of the shaft $(10,12 \mathrm{~cm})$, and the three types of stimulus configuration (baseline with vertical ends; leftward outgoing/rightward ingoing fins; leftward ingoing/rightward outgoing fins) generated six possible stimuli. Each type of stimulus was presented eight times, for a total of 48 trials. For each participant and for each session, a different random sequence was used. Two practice trials, one baseline, and one illusory stimulus selected at random, were administered at the beginning of each session (visual, haptic, visuo-haptic), and were not included in any subsequent analyses. 
After each response, using a ruler, the experimenter measured to the nearest millimeter the bisection error, namely, the distance between the subjective mid-point set by each participant and the objective center of the shaft. A rightward deviation of the subjective mid-point carried a plus sign, a leftward deviation a minus sign. A percent error as related to the length of each shaft (10, $12 \mathrm{~cm}$ ) was subsequently computed.

In order to assess the presence of USN in each sensory modality, percent errors of the baseline bisection task were first submitted to three analyses of variance (ANOVA), one per Presentation Modality, with one within-subjects main factor (Shaft Length: 10,12 cm), and one between-subjects main factor (Group: N+, N-, C).

Subsequently, for each participant, average percent errors in each illusory stimulus condition were corrected for the average percent error in the baseline stimulus. Illusory effects were investigated by three separate ANOVAs (one per Presentation Modality) with two within-subjects main factors (Stimulus: leftward outgoing/rightward ingoing fins, leftward ingoing/rightward outgoing fins; Shaft Length: 10, $12 \mathrm{~cm}$ ), and one between-subjects main factor (Group: $\mathrm{N}+, \mathrm{N}-, \mathrm{C})$. A posteriori contrasts among means were evaluated by Scheffé's test.

\section{RESULTS \\ BASELINE ERRORS}

The bisection of the baseline stimulus (a shaft with vertical ends) was deviated rightward in the $\mathrm{N}+$ group in the visual modality, indicating the presence of visual USN. No difference across the three groups was found under unimodal haptic and cross modal visuo-haptic presentations (see Figure 3).

In the visual condition, the analysis of variance revealed a significant main effect of Group $[F(2,25)=20.32, p<0.0001$, $\left.\eta^{2}=0.399\right]$. The main effect of Shaft Length was significant $\left[F(1,25)=5.31, p=0.030, \eta^{2}=0.161\right]$, indicating larger rightward errors with $12 \mathrm{~cm}$ than with $10 \mathrm{~cm}$ stimuli. The interaction Shaft Length by Group was not significant $[F(2,25)=1.32$, $\left.p=0.285, \eta^{2}=0.080\right]$. The differences between $\mathrm{N}+$ patients and both $\mathrm{N}-(p<0.0001)$ and $\mathrm{C}$ participants $(p<0.0001)$ were significant; no difference was found between the $\mathrm{N}-$ and $\mathrm{C}$ groups.

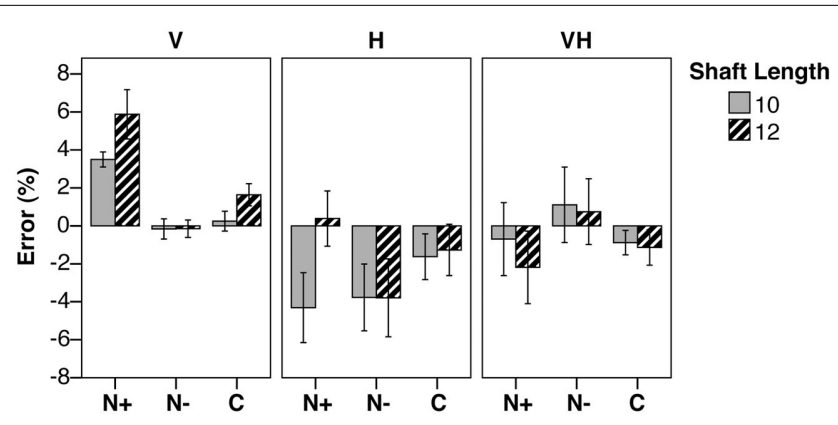

FIGURE 3 | Baseline errors. Mean percent error ( \pm SEM) in shaft bisection in the baseline condition, by Shaft Length $(10,12 \mathrm{~cm})$, Group $(\mathrm{N}+/ \mathrm{N}-$, patients with/without USN; C, neurologically unimpaired control participants), and presentation Modality (Visual, V; Haptic, H, Visuo-Haptic, VH). Negative/positive score: leftward/rightward error.
In the haptic condition, the main effect of Group was not significant $(F<1)$. The main effect of Shaft Length was significant $\left[F(1,25)=6.09, p=0.021, \eta^{2}=0.151\right]$, since the $10 \mathrm{~cm}$ stimulus was bisected more leftward than the $12 \mathrm{~cm}$ one. The interaction Shaft Length by Group was significant $[F(2,25)=4.55$, $\left.p=0.021, \eta^{2}=0.226\right]$. The difference between short and long stimuli was significant only in the $\mathrm{N}+$ group $(p=0.001)$ : in particular, $\mathrm{N}+$ patients bisected the longer rod more rightward than the shorter one.

In the visuo-haptic condition, the main effect of Group was not significant $(F<1)$. Also the main effect of Shaft Length $\left[F(1,25)=1.13, p=0.298, \eta^{2}=0.042\right]$ and its interaction with Group $(F<1)$ were not significant.

\section{ILLUSORY EFFECTS}

In each modality and in each group, stimuli with leftward outgoing/rightward ingoing fins brought about a leftward error, stimuli with leftward ingoing/rightward outgoing fins elicited a rightward error (Figure 4). The figure does not show the effect of Shaft Length, which did not provide results of interest for the purposes of the present study.

In the visual condition, the analysis of variance did not reveal a significant main effect of Group $[F(2,25)=2.51, p=0.101$, $\left.\eta^{2}=0.148\right]$; the main effect of Stimulus $[F(1,25)=138.88$, $\left.p<0.0001, \eta^{2}=0.772\right]$, and its interaction with the main effect of Shaft Length $\left[F(1,25)=6.60, p=0.017, \eta^{2}=0.004\right]$ were significant. Post hoc comparisons did not show any significant difference between the two lengths for both the leftward outgoing/rightward ingoing $(p=0.322)$, and the leftward ingoing/rightward outgoing stimuli $(p=0.236)$. The main effect of Shaft Length and all the interactions with Group were not significant $(F<1)$.

In the haptic condition, there was a trend toward significance for the main effect of Group $[F(2,25)=3.11, p=0.062$, $\left.\eta^{2}=0.182\right]$. N- patients bisected the stimuli overall more rightward than both the $\mathrm{N}+(p=0.035)$ and the $\mathrm{C}(p=0.037)$ participants. The main effect of Stimulus $[F(1,25)=45.663, p<0.0001$, $\left.\eta^{2}=0.501\right]$ and its interaction with the main effect of Shaft Length $\left[F(1,25)=4.96, p=0.035, \eta^{2}=0.006\right]$ were significant.

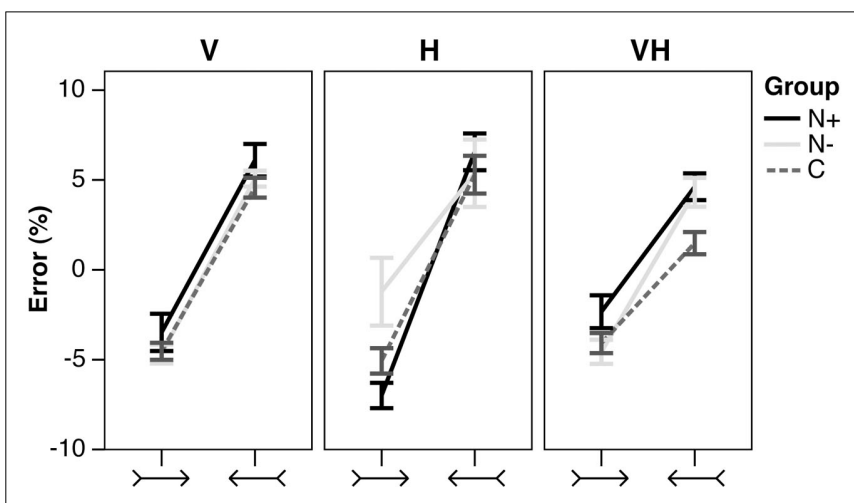

FIGURE 4 | Illusory effects. Mean percent error ( \pm SEM) in shaft bisection in the illusory conditions, by Stimulus type (leftward outgoing/rightward ingoing, and leftward ingoing/rightward outgoing fins), Group $(\mathrm{N}+, \mathrm{N}-, \mathrm{C})$, and presentation Modality $(\mathrm{V}, \mathrm{H}, \mathrm{VH})$. Negative/positive score: leftward/rightward error. 
The difference between the two lengths was close to significance in the leftward ingoing/rightward outgoing Stimulus $(p=0.076)$. The main effect of Shaft Length and its interaction with the main effect of Group was not significant $(F<1)$. The Stimulus by Group $\left[F(2,25)=1.50, p=0.243, \eta^{2}=0.033\right]$, and Stimulus by Length by Group $\left[F(2,25)=1.43, p=0.258, \eta^{2}=0.003\right]$ interactions were not significant.

Finally, in the visuo-haptic condition, the main effect of Group was significant $\left[F(2,25)=5.92, p=0.008, \eta^{2}=0.321\right]$. The difference between the $\mathrm{N}+$ and the $\mathrm{C}$ groups was significant $(p<0.009)$ : the corrected bisection of the illusory stimuli made by $\mathrm{N}+$ patients was shifted overall more rightward than the bisection made by $C$ participants. No other difference was significant. The main effect of Stimulus $\left[F(1,25)=113.01, p<0.0001, \eta^{2}=0.622\right]$ was significant. The main effect of Shaft Length $(F<1)$, and the Stimulus by Group $\left[F(2,25)=2.18, p=0.123, \eta^{2}=0.025\right]$, Stimulus by Shaft Length $\left[F(1,25)=1.68, p=0.206, \eta^{2}=0.004\right]$, Shaft Length by Group $(F<1)$, and Stimulus by Shaft Length by Group $\left[F(2,25)=1.36, p=0.274, \eta^{2}=0.007\right]$ interactions were not significant.

\section{DISCUSSION}

The present study yielded three main findings. (1) The rightward bias in bisection characteristic of patients with USN was present only in the visual modality, not in the haptic and visuo-haptic conditions. (2) Illusory effects were preserved in each modality, revealing that the processing of the illusion is independent of the presence of spatial attentional deficits. (3) Visual and tactile inputs were properly integrated by RBD patients with left USN.

\section{USN WITHIN AND BETWEEN SENSORY MODALITIES}

Our results support the view that left USN can be modalityspecific. In the present study, USN (as evaluated by a bisection task) was present in the visual modality only, since USN patients showed a preserved performance in the haptic and cross modal presentation conditions. It may be noted, however, that in the haptic modality USN patients did make a more rightward error with the longer stimulus (Figure 3), unlike patients without USN and control participants. This pattern might be taken as possible evidence of a minor tactile USN. In the visuo-haptic condition (visual illusion and haptic line bisection), illusory effects were biased rightwards in USN patients as compared with control participants (Figure 4), possibly indicating transfer of the visual USN in the tactile domain.

Overall, these results are in line with the evidence that USN is absent or less severe in the tactile than in the visual modality: a number of studies reports almost preserved tactile bisection in patients with visual USN (Fujii et al., 1991; Hjaltason et al., 1993; Chokron et al., 2002). We cannot exclude that in the present experiment the use of short rods $(<12 \mathrm{~cm})$ might have hidden deficits in haptic bisection: particularly, this is suggested by the fact that USN patients show a small rightward bias (see Figure 3) in the haptic bisection of longer $(12 \mathrm{~cm})$, but not of smaller rods $(10 \mathrm{~cm})$. However, longer rods have been used in previous studies that do not report a greater rightward error in haptic line bisection in USN patients than in control participants (Fujii et al., 1991, four lengths from 8 to $20 \mathrm{~cm}$ in $4 \mathrm{~cm}$ steps; Hjaltason et al., 1993,
20, and $40 \mathrm{~cm}$; Chokron et al., 2002, 20, and $22 \mathrm{~cm}$ ). Interestingly, the experiment of Hjaltason et al. (1993) includes a visuo-tactile task, in which participants indicate the perceived mid-point after having ran the index finger along the rod. Even in that condition (which differs from the visual one in that tactile exploration of the rod is required), RBD patients with left USN show no significant rightward error. In our visuo-haptic condition, the rod to bisect is explored only haptically, and participants are allowed to see its ends.

It is possible that our selection criteria for USN, based on visual tasks, may have prevented the inclusion of patients with haptic USN, at least in part accounting for the present results in terms of selection bias. However, it should be noted that previous studies reporting the absence of USN in haptic line bisection adopted similar selection criteria, namely the presence of visual USN (Fujii et al., 1991; Hjaltason et al., 1993; Chokron et al., 2002). In any case, in the baseline experimental task we assessed visual and haptic (as well as visuo-haptic) line bisection, replicating the previously reported visuo-haptic dissociation.

The rightward bias exhibited by RBD USN patients in visual bisection can not be traced back to initial rightward biases or to a general position preference for the side ipsilateral to the side of the lesion (ipsilesional; Campbell and Oxbury, 1976; Costa, 1976), since the shafts are short $(10$ and $12 \mathrm{~cm})$ and the participants' open hand covered the whole stimulus at the beginning of each trial.

Multisensory interactions have been rarely investigated in USN. One area of research involves the effects of physiological stimulations that improve a number of manifestations of the USN syndrome (Vallar et al., 1997; Kerkhoff, 2003; Rode et al., 2006a; Chokron et al., 2007). Vestibular stimulation ameliorates left-sided somatosensory deficits (Vallar et al., 1990, 1993b; Bottini et al., 2005). Prism adaptation improves tactile deficits (Maravita et al., 2003). Optokinetic stimulation may either ameliorate or worsen proprioceptive deficits of position sense in RBD patients with left USN (Vallar et al., 1993a, 1995a). Also auditory USN, as indexed by a contralesional left-sided deficit with dichotic stimuli, is ameliorated by prism adaptation (Jacquin-Courtois et al., 2010). The effects of these stimulations are essentially similar across sensory modalities and depend on the characteristics of the sensory stimulations (e.g., side, left vs. right, and type, warm vs. cold stimulation, in the case of caloric vestibular stimulation: see reviews in Vallar et al., 1997; Kerkhoff, 2003; Rode et al., 2006a; Chokron et al., 2007). Importantly, these stimulations are thought to modulate spatial processing or attention (biased ipsilaterally in USN patients), rather than being considered as an index of preserved multisensory integration. In particular, the typical paradigm of these studies involves the assessment of the patients' performance in unimodal tasks.

More direct evidence comes from the finding that in RBD patients the detection of contralesional visual stimuli is improved by the concomitant presentation of stimuli in another sensory modality (i.e., auditory), depending on the temporal and spatial coincidence of the sensory inputs (Frassinetti et al., 2002b, 2005). Differently from the effects of the sensory stimulations discussed above, these effects have been interpreted as based on the 
integrative contribution of multisensory neurons and neural networks, spared in patients with USN (Stein and Stanford, 2008). In particular, since these cross modal effects have been found both in patients with sensory deficits (namely, hemianopia) and with visuo-spatial deficits (namely, USN), the underlying mechanisms might involve preserved multisensory integration, with effects similar to those found in neurologically unimpaired participants (Frassinetti et al., 2002a; Bolognini et al., 2005). These studies investigated the multisensory integration of multiple sources of information about the same stimulus, i.e., coincident visual and auditory targets. A different form of integration is the combination between non-redundant sensory inputs, necessary to form a robust and coherent representation (Ernst and Bulthoff, 2004). The present study aimed specifically at investigating whether multisensory combination is preserved in USN, using the transfer of the illusion from vision to haptics as an index of effective integration.

The present results suggest that multisensory combinations, over or above contralesional unimodal sensory deficits, are preserved in RBD patients with USN, and are therefore independent of the presence of spatial deficits. This conclusion is further supported by two findings: first, patients exhibit visual but not, or minor, haptic USN; second, illusory effects are not modalityspecific. Interestingly, in the visuo-haptic condition (Figure 4), the finding that $\mathrm{N}+$ patients show a greater overall rightward bias with respect to control participants may be taken as a further evidence of visuo-haptic integration, as if visual USN cross modally affected tactile bisection. The preserved multisensory interactions reported here do not extend to other haptic processes, such as shape recognition, that was defective in four out of seven $\mathrm{N}+$ patients and in one out of seven $\mathrm{N}-$ patients. Overall, shape recognition and cross modal integration appear to rely on largely independent processes, which, in turn, do not involve the spatial attentional resources defective in USN.

Consequently, results from the present visuo-haptic paradigm do not lend support to the "amorphosynthesis" hypothesis of Denny-Brown et al. (1952), even though this account was formulated in rather vague terms. More recently, Brandt et al. (2009) proposed that USN reflects the damage of a multisensory integration center for attention and orientation (MSO) in the temporoparietal cortex. The MSO is assumed to be bilateral, but the center localized in the right hemisphere is held to be dominant, in that it exerts a greater inhibition of the contralateral left MSO, and a greater excitation of the ipsilateral visual cortex. The net result is that a right-sided temporo-parietal lesion of the MSO brings about visual USN mainly through a reduced activity of the right-sided visual cortex, which is further inhibited by the contralateral visual cortex. This model considers USN mainly as a visual phenomenon, and therefore could seem in accordance with the present results at first analysis. However, USN has been found also in the tactile (Vallar et al., 1991a, 1993b; Smania and Aglioti, 1995) and auditory (Bisiach et al., 1984; Vallar et al., 1995b; Jacquin-Courtois et al., 2010) modalities, in line with the multi-componential nature of the disorder (Vallar, 1998). Also, the multisensory integration features of the MSO center do not appear supported by the present results, which clearly reveal preserved visuo-haptic interactions in RBD patients with left USN.

\section{PROCESSING OF THE JUDD ILLUSION}

The illusory effects are preserved in each sensory condition, and independent of the presence of USN. Preserved leftward illusory effects have been already demonstrated in the visual modality, using variants of the Müller-Lyer figure (see Daini et al., 2002; Vallar and Daini, 2006, for reviews). These findings are in striking contrast with the evidence that the explicit processing of the left-sided portion of such stimuli is defective, as assessed by the verbal report of left-sided fins (Mattingley et al., 1995) and by same-different judgments (Ro and Rafal, 1996; Olk et al., 2001). Here we demonstrate for the first time that also tactile and cross modal illusory effects are preserved in RBD patients with left visual USN. Therefore, the Judd illusion can be a powerful tool for evaluating multimodal visual, haptic, and cross modal processes.

The present findings provide a definite indication that the spatial and attentional resources disrupted by USN do not play an important role in the processing of illusions such as the Judd variant of the Müller-Lyer figure. These processes may be largely non-spatial in nature, with the illusion eliciting a bias in mechanisms involved in cross modal shape representation in ventral stream networks (Vallar and Mancini, 2010; Mancini et al., 2011). A recent study from our laboratory indicates that a region in the extra-striate visual cortex, the lateral occipital complex, is a crucial underpinning of the multisensory Judd illusion (Mancini et al., 2011). This region is not a lesional correlate of USN, as assessed by line bisection tasks. In our patients the cortico-subcortical lesions mainly involve frontal and centro-parietal regions, relatively sparing the extra-striate visual cortex (see Figure 1). Anatomo-clinical correlation studies in RBD patients with USN show that the rightward bias in line bisection is associated with posterior lesions (Binder et al., 1992): specifically, to the inferior parietal lobule (Mort et al., 2003; Verdon et al., 2010), and at the temporo-occipital junction (Rorden et al., 2006).

In the present study the illusory effects were assessed by a line bisection task. This method, which provides a measure of the Müller-Lyer and related illusions, has been largely used in neuropsychological investigations of the processing of illusory visual stimuli (review in Vallar and Daini, 2006), and specifically using the Judd illusion, in previous studies from our laboratory (Mancini et al., 2010, 2011). In studies performed in neurologically unimpaired participants other response effectors have been investigated, such as saccadic eye movements, visually guided pointing, and grasping, with different results. Saccades are biased by the illusion, suggesting no dissociation between this type of action and perception (see the meta analysis of Bruno et al., 2010). As for visual effects, the prediction could be made that USN patients, being sensitive to illusory effects as assessed by line bisection, would exhibit a modulation of saccades by illusory stimuli. Beside saccades, the visual illusion can also affect grasping movements (Bruno and Franz, 2009). Conversely, visually guided pointing is not affected by the illusion (see the meta analysis of Bruno et al., 2008). This finding is taken as broadly consistent with the distinction between vision-for-perception and vision-for-action (Milner and Goodale, 2006; Gangopadhyay et al., 2010), with the illusion arising in the visual perceptual ventral stream and not affecting the vision-foraction dorsal stream. However, the positive effects on saccades and 
grasping "do not appear to support independent spatial representations for vision-for-action and vision-for-perception" (Bruno and Franz, 2009).

\section{CLINICAL IMPLICATIONS}

USN includes both perceptual and premotor components, with the former involving a defective conscious spatial representation of sensory and internally generated events in the contralesional side, the latter an impairment in performing movements in a contralesional direction ("directional hypokinesia"), and a general ipsilesional bias (Vallar and Mancini, 2010). The present study did not aim at disentangling perceptual and premotor components of USN. This would require specific paradigms, which should contrast perception and action in a more or less compatible way. Nevertheless, we believe that the rightward bias we found in the visual bisection of baseline stimuli is likely to be mainly perceptual in nature for three main reasons. (i) The stimuli (10 and $12 \mathrm{~cm}$ in length) fit comfortably into the participants' hand, which was placed over the stimulus at the beginning of the trial, thus minimizing the need of manual exploration. (ii) The preserved illusory effects, as assessed by manual line bisection, involve both rightward and leftward shifts, performed by the unaffected right hand. (iii) Premotor pathological mechanisms appear to be less frequent

\section{REFERENCES}

Albert, M. L. (1973). A simple test of visual neglect. Neurology 23, 658-664.

Barbieri, C., and De Renzi, E. (1989). Patterns of neglect dissociation. Behav. Neurol. 2, 13-24.

Benton, A. L. (1994). Neuropsychological assessment. Annu. Rev. Psychol. 45, 1-23.

Beschin, N., Cazzani, M., Cubelli, R., Della Sala, S., and Spinazzola, L. (1996). Ignoring left and far: an investigation of tactile neglect. $\mathrm{Neu}$ ropsychologia 34, 41-49.

Binder, J., Marshall, R., Lazar, R., Benjamin, J., and Mohr, J. P. (1992). Distinct syndromes of hemineglect. Arch. Neurol. 49, 1187-1194.

Bisiach, E., Bulgarelli, C., Sterzi, R., and Vallar, G. (1983). Line bisection and cognitive plasticity of unilateral neglect of space. Brain Cogn. 2, 32-38.

Bisiach, E., Capitani, E., Colombo, A., and Spinnler, H. (1976). Halving a horizontal segment: a study on hemisphere-damaged patients with cerebral focal lesions. Schweiz. Arch. Neurol. Psychiatr. 118, 199-206.

Bisiach, E., Cornacchia, L., Sterzi, R., and Vallar, G. (1984). Disorders of perceived auditory lateralization after lesions of the right hemisphere. Brain 107, 37-52.

Bisiach, E., and Faglioni, P. (1974). Recognition of random shapes by patients with unilateral lesions as a function of complexity, association value and delay. Cortex 10, 101-110. Bolognini, N., Frassinetti, F., Serino, A., and Làdavas, E. (2005). "Acoustical vision" of below threshold stimuli: interaction among spatially converging audiovisual inputs. Exp. Brain Res. 160, 273-282.

Bottini, G., Paulesu, E., Gandola, M., Loffredo, S., Scarpa, P., Sterzi, R., Santilli, I., Defanti, C. A., Scialfa, G., Fazio, F., and Vallar, G. (2005). Left caloric vestibular stimulation ameliorates right hemianesthesia. $\mathrm{Neu}$ rology 65, 1278-1283.

Brandt, T., Glasauer, S., Strupp, M., and Dieterich, M. (2009). Spatial neglect: hypothetical mechanisms of disturbed interhemispheric crosstalk for orientation. Ann. N. Y. Acad. Sci. 1164, 216-221.

Brozzoli, C., Demattè, M. L., Pavani, F., Frassinetti, F., and Farnè, A. (2006). Neglect and extinction: within and between sensory modalities. Restor. Neurol. Neurosci. 24, 217-232.

Bruno, N., Bernardis, P., and Gentilucci, M. (2008). Visually guided pointing, the Müller-Lyer illusion, and the functional interpretation of the dorsal-ventral split: conclusions from 33 independent studies. Neurosci. Biobehav. Rev. 32, 423-437.

Bruno, N., and Franz, V. H. (2009). When is grasping affected by the Müller-Lyer illusion? A quantitative review. Neuropsychologia 47, 1421-1433.

determinants of USN than the perceptual deranged components (Gallace et al., 2008; Vallar and Mancini, 2010).

Finally, on a clinical note, the present findings that USN can be absent in the tactile domain and spare cross modal interactions support the importance of including a multimodal assessment in diagnostic batteries, and of setting up multisensory-based rehabilitation approaches rather than the traditional visual treatments (Pizzamiglio et al., 2006; Schroder et al., 2008). The most important functions of multisensory integration are likely to be maximizing information delivered from the different sensory modalities, reducing the variance in the multisensory sensory estimate, in order to increase its reliability (Ernst and Bulthoff, 2004). Left USN may cause a bias in one modality, but the brain can take advantage of other preserved sensory modalities to help correcting it. Treatments that support these processes should be encouraged.

\section{ACKNOWLEDGMENTS}

This work has been supported in part by University of MilanoBicocca FAR grants to Emanuela Bricolo and Giuseppe Vallar, and by Ricerca Corrente grants to Giuseppe Vallar, from the Italian Auxological Institute, Milan, Italy. Flavia Mancini has been supported by a doctoral program scholarship of the University of Milano-Bicocca, Italy.

Bruno, N., Knox, P. C., and De Grave, D. D. (2010). A metanalysis of the effect of the Müller-Lyer illusion on saccadic eye movements: no general support for a dissociation of perception and oculomotor action. Vision Res. 50, 2671-2682.

Campbell, D. C., and Oxbury, J. M. (1976). Recovery from unilateral visuo-spatial neglect? Cortex 12, 303-312.

Chedru, F. (1976). Space representation in unilateral spatial neglect. J. Neurol. Neurosurg. Psychiatr. 39, 1057-1061.

Chokron, S., Colliot, P., Bartolomeo, P. Rhein, F., Eusop, E., Vassel, P., and Ohlmann, T. (2002). Visual, proprioceptive and tactile performance in left neglect patients. Neuropsychologia 40, 1965-1976.

Chokron, S., Dupierrix, E., Tabert, M. and Bartolomeo, P. (2007). Experimental remission of unilateral spatial neglect. Neuropsychologia 45, 3127-3148.

Costa, L. D. (1976). Interset variability on the raven coloured progressive matrices as an indicator of specific ability deficit in brain-lesioned patients. Cortex 12, 31-40.

Crawford, J. R., and Garthwaite, P. H. (2002). Investigation of the single case in neuropsychology: confidence limits on the abnormality of test scores and test score differences. Neuropsychologia 40, 1196-1208.

Cubelli, R., Nichelli, P., Bonito, V., De Tanti, A., and Inzaghi, M. G. (1991).
Different patterns of dissociation in unilateral spatial neglect. Brain Cogn. 15, 139-159.

Daini, R., Angelelli, P., Antonucci, G., Cappa, S. F., and Vallar, G. (2002). Exploring the syndrome of spatial unilateral neglect through an illusion of length. Exp. Brain Res. 144, 224-237.

De Renzi, E., Faglioni, P., and Scotti, G. (1970). Hemispheric contribution to exploration of space through the visual and tactile modality. Cortex 6 , 191-203.

Denny-Brown, D., Meyer, J. S., and Horenstein, S. (1952). The significance of perceptual rivalry resulting from parietal lesion. Brain 75, 433-471.

Diller, L., and Weinberg, J. (1977). "Hemi-inattention in rehabilitation. The evolution of a rational remediation program," in Hemi-inattention and Hemisphere Specialization, eds E. A. Weinstein and R. P. Friedland (New York: Raven Press), 62-82.

Ernst, M. O., and Bulthoff, H. H. (2004). Merging the senses into a robust percept. Trends Cogn. Sci. (Regul. Ed.) 8, 162-169.

Fortis, P., Maravita, A., Gallucci, M., Ronchi, R., Grassi, E., Senna, I., Olgiati, E., Perucca, L., Banco, E., Posteraro, L., Tesio, L., and Vallar, G. (2010). Rehabilitating patients with left spatial neglect by prism exposure during a visuomotor activity. Neuropsychology 24, 681-697. 
Frassinetti, F., Bolognini, N., Bottari, D., Bonora, A., and Làdavas, E. (2005). Audiovisual integration in patients with visual deficit. J. Cogn. Neurosci. 17, 1442-1452.

Frassinetti, F., Bolognini, N., and Làdavas, E. (2002a). Enhancement of visual perception by crossmodal visuo-auditory interaction. Exp. Brain Res. 147, 332-343.

Frassinetti, F., Pavani, F., and Làdavas, E. (2002b). Acoustical vision of neglected stimuli: interaction among spatially converging audiovisual inputs in neglect patients. $J$. Cogn. Neurosci. 14, 62-69.

Fujii, T., Fukatsu, R., Kimura, I., Saso, S., and Kogure, K. (1991). Unilateral spatial neglect in visual and tactile modalities. Cortex 27, 339-343.

Gainotti, G. (2010). The role of automatic orienting of attention towards ipsilesional stimuli in non-visual (tactile and auditory) neglect: a critical review. Cortex 46, 150-160.

Gainotti, G., Messerli, P., and Tissot, R. (1972). Qualitative analysis of unilateral spatial neglect in relation to laterality of cerebral lesions. J. Neurol. Neurosurg. Psychiatr. 35, 545-550.

Gallace, A., Imbornone, E., and Vallar, G. (2008). When the whole is more than the sum of the parts: evidence from visuospatial neglect. J. Neuropsychol. 2, 387-413.

Gangopadhyay, N., Madary, M., and Spicer, F. (2010). Perception, Action and Consciousness. Sensorimotor Dynamics and the Two Visual Systems. Oxford: Oxford University Press.

Gauthier, L., Dehaut, F., and Joanette, Y. (1989). The bells test: a quantitative and qualitative test for visual neglect. Int. J. Clin. Neuropsychol. 11, 49-54.

Gentilini, M., Barbieri, C., De Renzi, E., and Faglioni, P. (1989). Space exploration with and without the aid of vision in hemisphere-damaged patients. Cortex 25, 643-651.

Gilliatt, R. W., and Pratt, R. T. (1952). Disorders of perception and performance in a case of right-sided cerebral thrombosis. J. Neurol. Neurosurg. Psychiatr. 15, 264-271.

Gottesman, R. F., Kleinman, J. T., Davis, C., Heidler-Gary, J., Newhart, M., Kannan, V., and Hillis, A. E. (2008). Unilateral neglect is more severe and common in older patients with right hemispheric stroke. Neurology 71, 1439-1444.

Grigoletto, F., Zappala, G., Anderson, D. W., and Lebowitz, B. D. (1999). Norms for the Mini-Mental State Examination in a healthy population. Neurology 53, 315-320.

Haeske-Dewick, H. C., Canavan, A. G., and Homberg, V. (1996). Directional hyperattention in tactile neglect within grasping space. J. Clin. Exp. Neuropsychol. 18, 724-732.

Halligan, P. W., Fink, G. R., Marshall, J. C., and Vallar, G. (2003). Spatial cognition: evidence from visual neglect. Trends Cogn. Sci. (Regul. Ed.) 7, 125-133.

Heilman, K. M., Watson, R. T., and Valenstein, E. (2003). "Neglect and related disorders," in Clinical $\mathrm{Neu}$ ropsychology, 4 Edn, eds K. M. Heilman and E. Valenstein (New York: Oxford University Press), 296-346.

Hier, D. B., Mondlock, J., and Caplan, L. R. (1983a). Behavioral abnormalities after right hemisphere stroke. Neurology 33, 337-344.

Hier, D. B., Mondlock, J., and Caplan, L. R. (1983b). Recovery of behavioral abnormalities after right hemisphere stroke. Neurology 33, 345-350.

Hillis, A. E., Chang, S., Heidler-Gary, J., Newhart, M., Kleinman, J. T., Davis, C., Barker, P. B., Aldrich, E., and Ken, L. (2006). Neural correlates of modality-specific spatial extinction. J. Cogn. Neurosci. 18, 1889-1898.

Hjaltason, H., Caneman, G., and Tegner, R. (1993). Visual and tactile rod bisection in unilateral neglect. Cortex 29, 583-588.

Holding, D. H. (1970). Notes and discussion. A line illusion with irrelevant depth cues. Am. J. Psychol. 83, 280-282.

Husain, M. (2008). "Hemispatial neglect," in Handbook of Clinical Neurology, eds P. J. Vinken and G. W. Bruyn (Amsterdam: Elsevier), 359-372.

Jacquin-Courtois, S., Rode, G., Pavani, F., O'shea, J., Giard, M. H., Boisson, D., and Rossetti, Y. (2010). Effect of prism adaptation on left dichotic listening deficit in neglect patients: glasses to hear better? Brain 133, 895-908.

Judd, C. H. (1899). A study of geometrical illusions. Psychol. Rev. 6, 241-261.

Kerkhoff, G. (2003). Modulation and rehabilitation of spatial neglect by sensory stimulation. Prog. Brain Res. 142, 257-271.

Làdavas, E., Menghini, G., and Umiltà, C. (1994). A rehabilitation study of hemispatial neglect. Cogn. Neuropsychol. 11, 75-95.

Leibovitch, F. S., Black, S. E., Caldwell, C. B., Ebert, P. L., Ehrlich, L. E., and Szalai, J. P. (1998). Brain-behavior correlations in hemispatial neglect using CT and SPECT: the Sunnybrook Stroke Study. Neurology 50, 901-908.

Mancini, F., Bolognini, N., Bricolo, E., and Vallar, G. (2011). Cross-modal processing in the occipito-temporal cortex: a TMS Study of the MüllerLyer illusion. J. Cogn. Neurosci. 23 , 1987-1997.

Mancini, F., Bricolo, E., and Vallar, G. (2010). Multisensory integration in the Müller-Lyer illusion: from vision to haptics. Q. J. Exp. Psychol. 63, 818-830.

Maravita, A., Mcneil, J., Malhotra, P., Greenwood, R., Husain, M., and Driver, J. (2003). Prism adaptation can improve contralesional tactile perception in neglect. Neurology 60 1829-1831.

Mattingley, J. B., Bradshaw, J. L., and Bradshaw, J. A. (1995). The effects of unilateral visuospatial neglect on perception of Müller-Lyer illusory figures. Perception 24, 415-433.

Milner, A. D., and Goodale, M. (2006). The Visual Brain in Action. Oxford: Oxford University Press.

Mort, D. J., Malhotra, P., Mannan, S K., Rorden, C., Pambakian, A., Kennard, C., and Husain, M. (2003). The anatomy of visual neglect. Brain 126, 1986-1997.

Olk, B., Harvey, M., Dow, L., and Murphy, P. J. (2001). Illusion processing in hemispatial neglect. Neuropsychologia 39, 611-625.

Orsini, A., Grossi, D., Capitani, E., Laiacona, M., Papagno, C., and Vallar, G. (1987). Verbal and spatial immediate memory span: normative data from 1355 adults and 1112 children. Ital. J. Neurol. Sci. 8 539-548.

Perani, D., Vallar, G., Cappa, S., Messa C., and Fazio, F. (1987). Aphasia and neglect after subcortical stroke. A clinical/cerebral perfusion correlation study. Brain 110, 1211-1229.

Pizzamiglio, L., Antonucci, G., Judica, A., Montenero, P., Razzano, C., and Zoccolotti, P. (1992). Cognitive rehabilitation of the hemineglect disorder in chronic patients with unilateral right brain damage. J. Clin. Exp. Neuropsychol. 14, 901-923.

Pizzamiglio, L., Guariglia, C., Antonucci, G., and Zoccolotti, P. (2006). Development of a rehabilitative program for unilateral neglect. Restor. Neurol. Neurosci. 24, 337-345.

Ro, T., and Rafal, R. D. (1996). Perception of geometric illusions in hemispatial neglect. Neuropsychologia 34 973-978.

Rode, G., Klos, T., Courtois-Jacquin, S. Rossetti, Y., and Pisella, L. (2006a). Neglect and prism adaptation: a new therapeutic tool for spatial cognition disorders. Restor. Neurol. Neurosci. 24, 347-356.

Rode, G., Michel, C., Rossetti, Y., Boisson, D., and Vallar, G. (2006b).
Left size distortion (hyperschematia) after right brain damage. $\mathrm{Neu}$ rology 67, 1801-1808.

Ronchi, R., Posteraro, L., Fortis, P., Bricolo, E., and Vallar, G. (2009). Perseveration in left spatial neglect: drawing and cancellation tasks. Cortex 45 , 300-312.

Rorden, C., and Brett, M. (2000). Stereotaxic display of brain lesions. Behav. Neurol. 12, 191-200.

Rorden, C., Fruhmann Berger, M., and Karnath, H. O. (2006). Disturbed line bisection is associated with posterior brain lesions. Brain Res. 1080, 17-25.

Schenkenberg, T., Bradford, D. C., and Ajax, E. T. (1980). Line bisection and unilateral visual neglect in patients with neurologic impairment. $\mathrm{Neu}$ rology 30, 509-517.

Schindler, I., Clavagnier, S., Karnath, H. O., Derex, L., and Perenin, M. T. (2006). A common basis for visual and tactile exploration deficits in spatial neglect? Neuropsychologia 44, 1444-1451.

Schroder, A., Wist, E. R., and Hömberg, V. (2008). TENS and optokinetic stimulation in neglect therapy after cerebrovascular accident: a randomized controlled study. Eur. J. Neurol. 15, 922-927.

Smania, N., and Aglioti, S. (1995). Sensory and spatial components of somaesthetic deficits following right brain damage. Neurology 45, 1725-1730.

Stein, B. E., and Stanford, T. R. (2008). Multisensory integration: current issues from the perspective of the single neuron. Nat. Rev. Neurosci. 9 , 255-266.

Vallar, G. (1998). Spatial hemineglect in humans. Trends Cogn. Sci. (Regul. Ed.) 2, 87-97.

Vallar, G. (2000). "The methodological foundations of human neuropsychology: studies in braindamaged patients," in Handbook of Neuropsychology, 2nd Edn, eds F. Boller, J. Grafman, and G. Rizzolatti (Amsterdam: Elsevier), 305-344.

Vallar, G., Antonucci, G., Guariglia, C., and Pizzamiglio, L. (1993a). Deficits of position sense, unilateral neglect and optokinetic stimulation. $\mathrm{Neu}$ ropsychologia 31, 1191-1200.

Vallar, G., Bottini, G., Rusconi, M. L., and Sterzi, R. (1993b). Exploring somatosensory hemineglect by vestibular stimulation. Brain 116, 71-86.

Vallar, G., Bottini, G., Sterzi, R., Passerini, D., and Rusconi, M. L. (1991a). Hemianesthesia, sensory neglect, and defective access to conscious experience. Neurology 41, 650-652. 
Vallar, G., Rusconi, M. L., Geminiani, G., Berti, A., and Cappa, S. F. (1991b). Visual and nonvisual neglect after unilateral brain lesions: modulation by visual input. Int. J. Neurosci. 61, 229-239.

Vallar, G., and Daini, R. (2006). "Visual perceptual processing in unilateral spatial neglect: the case of visual illusions," in Imagery and Spatial Cognition: Methods, Models and Cognitive Assessment, eds T. Vecchi and G. Bottini (Amsterdam/Philadelphia: John Benjamins Publishing Company), 337-362.

Vallar, G., Guariglia, C., Magnotti, L., and Pizzamiglio, L. (1995a). Optokinetic stimulation affects both vertical and horizontal deficits of position sense in unilateral neglect. Cortex 31, 669-683.

Vallar, G., Guariglia, C., Nico, D., and Bisiach, E. (1995b). Spatial hemineglect in back space. Brain 118 , 467-472.
Vallar, G., Guariglia, C., and Rusconi, M. L. (1997). "Modulation of the neglect syndrome by sensory stimulation," in Parietal Lobe Contributions to Orientation in $3 D$ Space, eds P. Thier and H.-O. Karnath (Heidelberg: Springer-Verlag), 555-578.

Vallar, G., and Mancini, F. (2010). "Mapping the neglect syndrome onto neurofunctional streams," in Perception, Action, and Consciousness. Sensorimotor Dynamics and Two Visual Systems, eds N. Gangopadhyay, M. Madary, and F. Spicer (Oxford: Oxford University Press), 183-215.

Vallar, G., Rusconi, M. L., Bignamini, L., Geminiani, G., and Perani, D. (1994a). Anatomical correlates of visual and tactile extinction in humans: a clinical CT scan study. J. Neurol. Neurosurg. Psychiatry 57, 464-470.

Vallar, G., Rusconi, M. L., Fontana, S. and Musicco, M. (1994b). Tre test di esplorazione visuo-spaziale: taratura su 212 soggetti normali. Arch. Psicol. Neurol. Psichiatr. 55, 827-841.

Vallar, G., Sterzi, R., Bottini, G., Cappa S., and Rusconi, M. L. (1990). Temporary remission of left hemianesthesia after vestibular stimulation. A sensory neglect phenomenon. Cor tex 26, 123-131.

Verdon, V., Schwartz, S., Lovblad, K. O., Hauert, C. A., and Vuilleumier, P. (2010). Neuroanatomy of hemispatial neglect and its functional components: a study using voxel-based lesion-symptom mapping. Brain 133, 880-894.

Villardita, C. (1987). Tactile exploration of space and visual neglect in braindamaged patients. J. Neurol. 234 292-297.

Conflict of Interest Statement: The authors declare that the research was conducted in the absence of any commercial or financial relationships that could be construed as a potential conflict of interest.

Received: 28 July 2011; accepted: 01 November 2011; published online: 22 November 2011.

Citation: Mancini F, Bricolo E, Mattioli FC and Vallar G (2011) Visuohaptic interactions in unilateral spatial neglect: the cross modal Judd illusion. Front. Psychology 2:341. doi: 10.3389/fpsyg.2011.00341

This article was submitted to Frontiers in Perception Science, a specialty of Frontiers in Psychology.

Copyright (C) 2011 Mancini, Bricolo, Mattioli and Vallar. This is an openaccess article subject to a non-exclusive license between the authors and Frontier Media SA, which permits use, distribution and reproduction in other forums, provided the original authors and source are credited and other Frontiers conditions are complied with. 